\title{
Effects of hypoglycaemia on working memory and regional cerebral blood flow in type 1 diabetes: a randomised, crossover trial
}

\author{
Michael Gej1 ${ }^{1,2}$ • Albert Gjedde ${ }^{3,4}$ - Birgitte Brock ${ }^{5,6}$ • Arne Møller ${ }^{1,7}$. \\ Eelco van Duinkerken ${ }^{8,9}$ • Hanne L. Haahr ${ }^{10}$ • Charlotte T. Hansen ${ }^{10}$. \\ Pei-Ling Chu ${ }^{11}$ • Kirstine L. Stender-Petersen ${ }^{10}$ • Jørgen Rungby ${ }^{1,2,12}$
}

Received: 14 June 2017 / Accepted: 3 October 2017 /Published online: 29 November 2017

(C) The Author(s) 2017. This article is an open access publication

\begin{abstract}
Aims/hypothesis The aim of this randomised, crossover trial was to compare cognitive functioning and associated brain activation patterns during hypoglycaemia (plasma glucose [PG] just below $3.1 \mathrm{mmol} / \mathrm{l}$ ) and euglycaemia in individuals with type 1 diabetes mellitus.
\end{abstract}

Electronic supplementary material The online version of this article (https://doi.org/10.1007/s00125-017-4502-1) contains peer-reviewed but unedited supplementary material, which is available to authorised users.

Michael Gejl

mg@biomed.au.dk

1 Department of Biomedicine, Aarhus University, Bartholins Allé 6 , Building 1242, 8000 Aarhus C, Denmark

2 Department of Endocrinology and Internal Medicine, Aarhus University Hospital, Aarhus, Denmark

3 Department of Neuroscience, University of Copenhagen, Copenhagen, Denmark

4 Department of Clinical Medicine, University of Southern Denmark, Odense, Denmark

5 Department of Clinical Biochemistry, Aarhus University Hospital, Aarhus, Denmark

6 Steno Diabetes Center Copenhagen, Gentofte, Denmark

7 PET-Center, Department of Nuclear Medicine, Aarhus University Hospital, Aarhus, Denmark

8 VU University Medical Centre, Amsterdam, the Netherlands

9 Pontifícia Universidade Católica, Rio de Janeiro, Brazil

10 Novo Nordisk A/S, Søborg, Denmark

11 Novo Nordisk Inc., Plainsboro, NJ, USA

12 Department of Endocrinology IC, Bispebjerg University Hospital, Bispebjerg, Copenhagen, Denmark
Methods In this patient-blinded, crossover study, 26 participants with type 1 diabetes mellitus attended two randomised experimental visits: one hypoglycaemic clamp (PG $2.8 \pm 0.2 \mathrm{mmol} / \mathrm{l}$, approximate duration $55 \mathrm{~min}$ ) and one euglycaemic clamp (PG $5.5 \mathrm{mmol} / \mathrm{l} \pm 10 \%)$. PG levels were maintained by hyperinsulinaemic glucose clamping. Cognitive functioning was assessed during hypoglycaemia and euglycaemia conditions using a modified version of the digit symbol substitution test (mDSST) and control DSST (cDSST). Simultaneously, regional cerebral blood flow (rCBF) was measured in pre-specified brain regions by six $\mathrm{H}_{2}{ }^{15} \mathrm{O}$-positron emission tomographies (PET) per session.

Results Working memory was impaired during hypoglycaemia as indicated by a statistically significantly lower mDSST score (estimated treatment difference [ETD] -0.63 [95\% CI -1.13 , $-0.14], p=0.014)$ and a statistically significantly longer response time (ETD $2.86 \mathrm{~s}$ [7\%] [95\% CI 0.67, 5.05], $p=0.013$ ) compared with euglycaemia. During hypoglycaemia, mDSST task performance was associated with increased activity in the frontal lobe regions, superior parietal lobe and thalamus, and decreased activity in the temporal lobe regions $(p<0.05)$. Working memory activation (mDSST - cDSST) statistically significantly increased blood flow in the striatum during hypoglycaemia (ETD $0.0374 \%$ [95\% CI 0.0157, 0.0590], $p=0.002$ ).

Conclusions/interpretation During hypoglycaemia (mean PG $2.9 \mathrm{mmol} / \mathrm{l}$ ), working memory performance was impaired. Altered performance was associated with significantly increased blood flow in the striatum, a part of the basal ganglia implicated in regulating motor functions, memory, language and emotion.

Trial registration NCT01789593, clinicaltrials.gov

Funding This study was funded by Novo Nordisk.

Keywords Clinical diabetes $\cdot$ Hypoglycaemia $\cdot$ Imaging (MRI/PET/other) · Insulin therapy 


$\begin{array}{ll}\text { Abbreviations } \\ \text { AE } & \text { Adverse event } \\ \text { CBF } & \text { Cerebral blood flow } \\ \text { cDSST } & \text { Control digit symbol substitution test } \\ \text { DSST } & \text { Digit symbol substitution test } \\ \text { ETD } & \text { Estimated treatment difference } \\ \text { fMRI } & \text { Functional MRI } \\ \text { mDSST } & \text { Modified digit symbol substitution test } \\ \text { PASAT } & \text { Paced auditory serial addition task } \\ \text { PET } & \text { Positron emission tomography } \\ \text { PG } & \text { Plasma glucose } \\ \text { rCBF } & \text { Regional cerebral blood flow } \\ \text { ROI } & \text { Regions of interest }\end{array}$

\section{Introduction}

Cognitive performance, even during simple tasks, is impaired during acute hypoglycaemia (plasma glucose $[\mathrm{PG}]<2.5 \mathrm{mmol} / 1$ [45.0 mg/dl]) in individuals with type 1 diabetes mellitus [1,2] and the degree of impairment depends on the level of hypoglycaemia [3]. Additionally, the cognitive impairment induced by hypoglycaemia (PG $2.5-2.7 \mathrm{mmol} / \mathrm{l}$ ) can remain following the restoration of euglycaemia [4-6]; with some studies showing cognitive impairments lasting for approximately 45-75 min after euglycaemic restoration [7, 8]. Working memory is an important aspect of cognitive function and is susceptible to the effects of hypoglycaemia. It has been demonstrated using functional MRI (fMRI) that, compared with healthy individuals, people with type 1 diabetes mellitus require higher levels of brain activation to attain parity for working memory performance during hypoglycaemia (PG $\leq 2.8 \mathrm{mmol} / 1[50.4 \mathrm{mg} / \mathrm{dl}]$ ) [9]. Thus, identification of cerebral activation patterns during working memory performance at different PG levels could enhance our understanding of mechanisms underlying the reduced cerebral efficiency seen in type 1 diabetes mellitus [9]. For example, it remains to be clarified if milder hypoglycaemic episodes, PG just below $3.1 \mathrm{mmol} / 1$ and previously associated with altered brain activity $[10,11]$, have a similar negative impact on working memory to those demonstrated at $\mathrm{PG} \leq 2.8 \mathrm{mmol} / \mathrm{l}$. In individuals with type 1 diabetes mellitus, cerebral blood flow (CBF) and cerebral glucose metabolism [12] within total grey matter are correlated. This correlation becomes stronger when adjusted for glucose levels, allowing CBF assessment to be used as a proxy for cerebral metabolism [13]. However, there are some limitations to assessment with fMRI; namely it does not provide a direct measure of neuronal oxygen consumption or neuronal activation and results are vulnerable to movement distortion. Conversely, radiolabelled water $\left(\mathrm{H}_{2}{ }^{15} \mathrm{O}\right)$ positron emission tomography (PET) is a direct measure of cerebral oxygen consumption (and thus neuronal activation) that is less affected by movement and more quantifiable than fMRI [14].
This hypothesis-driven study aimed to test if cognitive performance (assessed by $\mathrm{H}_{2}{ }^{15} \mathrm{O}$ PET) and associated CBF estimates are affected at less pronounced levels of hypoglycaemia than previously studied and if cognitive performance is affected in the recovery phase following less pronounced hypoglycaemia.

\section{Methods}

\section{Study design}

This randomised, single-centre, patient-blinded, two-period crossover study compared cognitive performance (assessed by working memory performance and reaction time) under hypoglycaemic (aiming for a PG target just below $3.1 \mathrm{mmol} /$; the clamp target was defined as $2.8 \pm 0.2[2.6-3.0] \mathrm{mmol} / \mathrm{l})$ and euglycaemic (PG clamp target $5.5 \mathrm{mmol} / \mathrm{l} \pm 10 \%$ ) conditions in participants with type 1 diabetes mellitus (ESM Fig. 1). Blood was drawn at pre-specified time points to assess counter-regulatory hormone responses. Hypoglycaemia awareness and symptoms during both clamps were also assessed. Participants underwent the sequence of glycaemic conditions in a blind and randomised order determined by sequential enrolment and lowest available number assignment. The two experimental visits were separated by $21-42$ days (to avoid effects of counter-regulatory hormone responses or other physiological effects of hypoglycaemia). Female participants attended the two visits at the same stage of their menstrual cycle. The study was conducted from 14 January to 1 December 2013 at the Department of Endocrinology and Department of Nuclear Medicine and PET Center, Aarhus University Hospital, Aarhus, Denmark. Informed written consent was obtained from all participants before any studyrelated activities. The study was conducted in compliance with International Conference on Harmonisation Good Clinical Practice [15], the Declaration of Helsinki [16] and was approved according to local regulations by an independent ethics committee.

\section{Participants}

Inclusion criteria for participants screened $(n=37)$ were righthandedness, age 18-64 years, BMI $18.0-28.0 \mathrm{~kg} / \mathrm{m}^{2}, \mathrm{HbA}_{1 \mathrm{c}}$ $\leq 9.0 \%$ ( $\leq 75 \mathrm{mmol} / \mathrm{mol})$, diagnosed with type 1 diabetes mellitus and treated with multiple daily insulin injections or continuous subcutaneous insulin infusion for $\geq 12$ months prior to screening. Key exclusion criteria included known central nervous system abnormalities, structural brain abnormalities (identified by structural MRI scans during screening), severe hypoglycaemia (requiring third party assistance) or ketoacidosis in the last 6 months, clinically defined hypoglycaemic unawareness, and treatment with medications potentially interfering with glucose metabolism. Key experimental visit exclusion 
criteria included occurrence of a hypoglycaemic event (with PG $\leq 3.9 \mathrm{mmol} / \mathrm{l}$ ) within the preceding $48 \mathrm{~h}$. Full inclusion and exclusion criteria are listed in ESM Table 1.

\section{Cognitive tests}

The Wechsler Adult Intelligence Scale (third edition) revised digit symbol substitution test (DSST) [17] was previously adapted and validated to specifically measure working memory [18], and has been successfully used with fMRI [19]. Here, we used the stimuli of this adapted DSST paradigm and modified it to our specific PET procedure. As with the fMRI design, we used the modified DSST (mDSST) task to measure working memory. A control task (cDSST) was used for non-task-related brain activation (without a working memory load), including visual and motor cortex activation (for eye sight and movement) and index finger movement.

Subtraction of regional $\mathrm{CBF}$ ( $\mathrm{rCBF}$ ) patterns during cDSST from those during mDSST was interpreted as indicative of brain activation patterns exclusively associated with the operation of working memory. The mDSST task consisted of three blocks of 32 randomly presented digitsymbol combinations (ESM Methods: Cognitive tests mDSST and cDSST task combination blocks and ESM Fig. 2), fitting the specific $\mathrm{H}_{2}{ }^{15} \mathrm{O}$ PET design. The cDSST task had the same basic design. Each block began with test instructions shown for $7.8 \mathrm{~s}$ and was $0.2 \mathrm{~s}$ longer than every $3 \mathrm{~min}$ PET acquisition, to ensure participants were engaged in the mDSST or cDSST task during the full 3 min PET acquisition. Tasks were presented using E-prime 2.0 (Psychology Software Tool, Pittsburgh, PA, USA) through audiovisual goggles inside the PET scanner. Correct responses (no response was considered as incorrect) and response time were recorded and analysed.

To test working memory following a prolonged recovery phase (75-90 min after PG of 5-6 $\mathrm{mmol} / \mathrm{l}$ was restored) following hypoglycaemia or euglycaemia, the paced auditory serial addition task (PASAT) was preferred over the mDSST to avoid bias from habituation [20, 21]. During the PASAT, participants heard a digit and had to add the next digit (presented 3 or 2 s later) and report the sum aloud. Both parts consisted of 60 digits and correct responses were recorded.

\section{PET imaging}

Each participant underwent PET imaging at both visits. First, a 6 min transmission scan for attenuation correction was performed. Thereafter, six 3 min tomography sessions with either mDSST or cDSST in a fixed order were performed. Cerebral activity levels were measured as change in brain uptake of radiolabelled water $\left(\mathrm{H}_{2}{ }^{15} \mathrm{O}\right)$, the retention of which matches the rate of $\mathrm{CBF}$, by means of a high-resolution research tomograph (Siemens/CTI, Knoxville, TN, USA) operating in 3-D mode. Additional detail is provided in ESM Methods: PET imaging.

\section{Experimental visit procedures}

Participants attended the study site at approximately 20:00 hours on the day before each experimental procedure, at which point normal insulin treatment was suspended. Participants stayed overnight to ensure stabilisation of $\mathrm{PG}$ within the range of 5-8 $\mathrm{mmol} / 1 \mathrm{via}$ variable intravenous infusion of insulin (Actrapid®, $100 \mathrm{U} / \mathrm{ml})$ and glucose $(20 \%$ glucose/dextrose/ $10 \mathrm{mmol} / \mathrm{KCl}$ ), before initiation of experimental procedures at 08:00 hours the following day. On the days of the experimental procedures, cognitive tests were briefly performed $\geq 1 \mathrm{~h}$ before initiation of hypoglycaemia or euglycaemia to prevent practice effects. Each glycaemic condition was preceded by a 60 min run-in period whereby variable intravenous infusions of glucose or human soluble insulin were delivered to obtain a steady-state PG target level of $5.5 \mathrm{mmol} / \mathrm{l} \pm 10 \%$. During the run-in and clamps, the participants' cannulated hand was placed in a thermoregulated box with their arterialised venous blood sampled for PG measurements using a benchtop glucose analyser (YSI 2300 Stat Plus, Yellow Springs, OH, USA). Euglycaemia was maintained using a glucose clamp (glucose/ Actrapid® infusion) for approximately $1 \mathrm{~h}$ after which insulin (Actrapid®, $100 \mathrm{U} / \mathrm{ml}$ ) was given at an infusion rate of $3 \mathrm{mU} \mathrm{kg}^{-1} \mathrm{~min}^{-1}$ for $10 \mathrm{~min}$ and then reduced to $1.5 \mathrm{mU} \mathrm{kg}^{-1} \mathrm{~min}^{-1}$ thereafter. Euglycaemia was maintained or hypoglycaemia induced with glucose infusion rate adjusted accordingly to meet the PG target for approximately $55 \mathrm{~min}$, during which $\mathrm{H}_{2}{ }^{15} \mathrm{O}$ PET scans and cDSST and mDSST tasks were performed (Fig. 1). Following glycaemic clamps, participants were brought back to euglycaemia (with glucose infusions to reach a PG target of 5-6 $\mathrm{mmol} / \mathrm{l}$ ) and after approximately 75-90 min the PASAT was conducted. After experimental procedures, participants resumed usual insulin treatment.

\section{Counter-regulatory hormones}

Counter-regulatory hormones were measured as a validation that the PG target was sufficient to elicit a counter-regulatory response, and thus, hypoglycaemia. Hormonal responses (noradrenaline [norepinephrine], glucagon, cortisol and growth hormones) were measured $30 \mathrm{~min}$ prior to induction of hypoglycaemia or euglycaemia, and $45 \mathrm{~min}$ and $150 \mathrm{~min}$ (just prior to PASAT test) after induction of hypoglycaemia or euglycaemia.

\section{Hypoglycaemia awareness and symptoms}

At screening, hypoglycaemia unawareness was assessed by asking participants 'Can you feel your hypos?' and checking 
Fig. 1 Experimental visit design

Variable glucose
infusion rate
$\begin{aligned} & \text { Insulin } \\ & \text { infusion rate }\end{aligned}$
PET scan
$\mathrm{PG}=5.5 \mathrm{mmol} / \mathrm{l}$
$\mathrm{PG}=2.8 \pm 0.2 \mathrm{mmol} / \mathrm{l}$
Cognitive testing
Blood sampling for
hormone response
Hypoglycaemia awareness
and symptoms

their medical files for any indication of unawareness. During the study, hypoglycaemia awareness was assessed both $30 \mathrm{~min}$ prior to and $45 \mathrm{~min}$ after induction of hypoglycaemia or euglycaemia, by asking participants 'Do you feel hypo?'. The hypoglycaemia symptoms questionnaire (based on the Edinburgh Condition Scale) [22] was completed by participants $30 \mathrm{~min}$ prior to and $55 \mathrm{~min}$ after induction of hypoglycaemia or euglycaemia. It measured autonomic (sweating, palpitations, shaking and hunger), neuroglycopenic (confusion, drowsiness, odd behaviour, speech difficulty and incoordination) and general malaise (headache and nausea) symptoms on a seven point Likert scale.

\section{rCBF assessment}

Cerebral activation was measured as $\mathrm{rCBF}$ in 19 pre-specified regions of interest (ROI). Given the hypothesis-driven nature of this study, these regions were selected in accordance with relevant literature for one or more of the following criteria: DSST evoked brain activity patterns in normal conditions, i.e. euglycaemia, without working memory load (precuneus, dorsolateral prefrontal cortex, anterior cingulate gyrus/cortex, posterior cingulate gyrus, posterior supramarginal gyrus and orbitofrontal cortex) [19, 23]; neural substrate of DSST performance (inferior frontal gyrus, superior frontal gyrus, middle frontal gyrus, superior parietal lobe, precuneus, posterior cingulate gyrus/cortex and parahippocampal gyrus) [24, 25]; brain areas showing changes in functional activity in response to hypoglycaemia (medial temporal lobe, hippocampus, parahippocampal gyrus, insula, globus pallidum, striatum, dorsolateral prefrontal cortex, anterior cingulate gyrus, inferior frontal gyrus, superior frontal gyrus, precuneus, posterior cingulate gyrus, posterior supramarginal gyrus and primary visual cortex) [9, 26-30]; and brain areas reported as involved in working memory tasks (dorsolateral prefrontal cortex, inferior
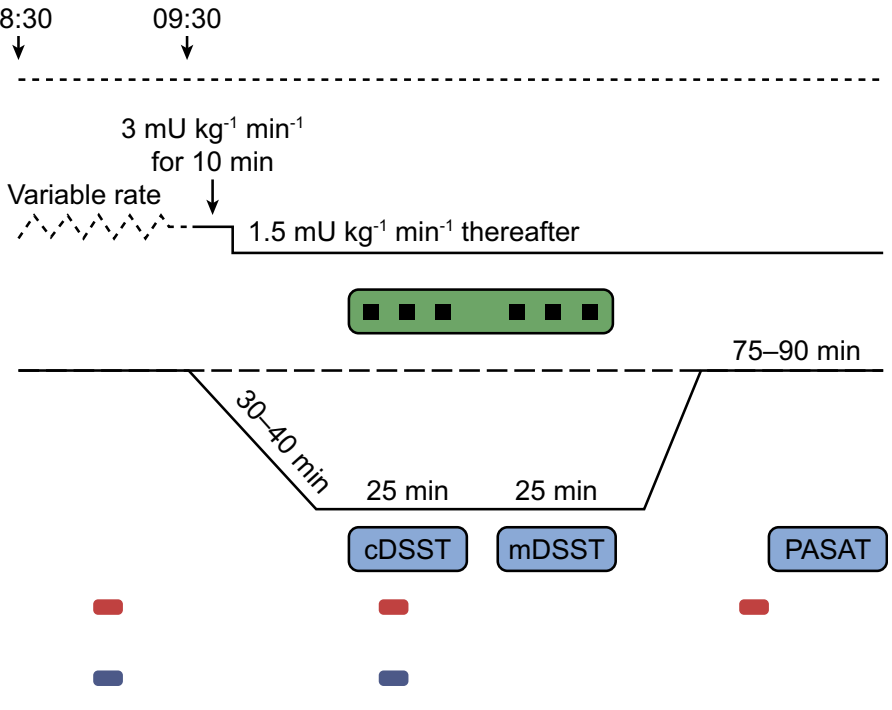

frontal gyrus, middle frontal gyrus, superior frontal gyrus, ventromedial prefrontal cortex, orbitofrontal cortex, insula, superior parietal lobe, anterior cingulate gyrus/cortex, hippocampus and thalamus) [31-33]. All rCBF measures were normalised to measures in the cerebral cortex, as this region is considered to be less impacted by the duration of type 1 diabetes mellitus [34]. In the present study, there was no significant difference in $\mathrm{rCBF}$ in the cerebral cortex between hypoglycaemia and euglycaemia during either cDSST or mDSST tasks.

Three endpoints were used to determine regional cerebral activation: $\mathrm{rCBF}$ during $\mathrm{mDSST}$ and cDSST performances, and $\mathrm{rCBF}$ for working memory. For the cDSST and mDSST endpoints, $\mathrm{rCBF}$ was calculated by subtracting the mean of three $\mathrm{rCBF}$ values for euglycaemia from the mean of three rCBF values for hypoglycaemia. To isolate changes as a result of working memory, rCBF values during the mDSST test were corrected for the $\mathrm{rCBF}$ values during cDSST, by subtracting the mean of three cDSST rCBF values from the mean of three mDSST rCBF values; this correction was conducted for measurements taken during both glycaemic clamps with the totals for euglycaemia subtracted from those for hypoglycaemia ([mean $(3 \times \mathrm{CBF}$ during $\mathrm{mDSST})-$ mean $(3$ $\times$ CBF during cDSST)] hypoglycaemia $-[$ mean $(3 \times \mathrm{CBF}$ during mDSST $)-$ mean $(3 \times \mathrm{CBF}$ during $\mathrm{cDSST})$ ] euglycaemia) to isolate changes in working memory during hypoglycaemia.

\section{Endpoints and statistical analyses}

The primary objective of the study was to compare cognitive performance during hypoglycaemia with that during euglycaemia. The primary endpoint was the number of correct mDSST scores. For each glycaemic condition, mean mDSST scores, reaction time and PASAT scores were compared using 
a linear mixed-effect model with glycaemic condition and period as fixed factors and participant as a random factor; mean differences between hypoglycaemia and euglycaemia were estimated from the model and corresponding 95\% CI and $p$ values were calculated. The $\mathrm{rCBF}$ and predefined ROI (during both cDSST and mDSST), as well as the difference in rCBF between the two tasks, were compared during glycaemic conditions using an analysis of variance with glycaemic condition, period and participant as fixed factors. Because of the hypothesis-driven nature of this trial, no correction for multiplicity was performed with regard to different ROI analyses.

The SD for DSST score between euglycaemia and hypoglycaemia (PG $2.5 \mathrm{mmol} / \mathrm{l}$ ) was determined in a previous trial (NCT01002768) to be approximately nine. Assuming a similar variability in this trial, using a $5 \%$ significance level and two-sided paired $t$ test, a sample size of 25 participants completing both periods was calculated to have $90 \%$ power to detect a true difference in DSST score between hypoglycaemia and euglycaemia of approximately six. A total of 28 participants were randomised to ensure at least 25 participants completing both experimental visits.

\section{Results}

\section{Participant disposition}

Of the 37 participants screened, 29 ( 22 men and seven women) were randomised and 26 completed both experimental visits (ESM Fig. 3); data are presented for completers only (Table 1).

Table 1 Baseline characteristics of completers

\begin{tabular}{ll}
\hline Characteristic & Value \\
\hline Number of participants & 26 \\
Age, years (mean $\pm \mathrm{SD})$ & $38.7 \pm 15.3$ \\
BMI, $\mathrm{kg} / \mathrm{m}^{2}$ (mean $\left.\pm \mathrm{SD}\right)$ & $24.6 \pm 2.7$ \\
Race & \\
$\quad$ Of European descent, $n(\%)$ & $26(100.0)$ \\
Sex & \\
$\quad$ Female, $n(\%)$ & $6(23.1)$ \\
$\quad$ Male, $n(\%)$ & $20(76.9)$ \\
Educational level, years & $14.0 \pm 2.0(10.0-18.0)$ \\
$\quad($ mean \pm SD $[$ min-max $])$ & \\
Diabetes characteristics & \\
$\quad($ mean $[$ min-max]) & \\
$\quad$ Duration of diabetes, years & $18.7(3.1-46.4)$ \\
$\quad$ HbA $_{1 \mathrm{c}}$, mmol/mol & $56.7(39.0-74.0)$ \\
$\quad$ HbA $_{1 \mathrm{c}}, \%$ & $7.3(5.7-8.9)$ \\
\hline
\end{tabular}

Baseline information was recorded at screening and/or randomisation. If an assessment was recorded on both visits, the randomisation value was used as the baseline value
Three participants were withdrawn after randomisation (two men and one woman) for inability to place the venous catheter $(n=1)$, erroneous randomisation $(n=1)$ and meeting experimental visit exclusion criteria $(n=1)$. Mean age of participants was 38.7 years (range 19.0-65.1 years), BMI was $24.6 \mathrm{~kg} / \mathrm{m}^{2}$, $\mathrm{HbA}_{1 \mathrm{c}}$ was $7.3 \%(56.7 \mathrm{mmol} / \mathrm{mol})$ and duration of diabetes was 18.7 years (Table 1). The educational level of participants was a mean $\pm \mathrm{SD}$ of $14.0 \pm 2.0$ years (Table 1). Individual PG profiles during hypoglycaemic and euglycaemic clamps are shown in ESM Fig. 4. Mean \pm SD PG achieved during hypoglycaemia was $2.9 \pm 0.14 \mathrm{mmol} / \mathrm{l}$, which is within the target PG. During the study four adverse events (AEs) were reported in three participants (two each during hypoglycaemia [presyncope and orthostatic hypotension] and euglycaemia [headache and nausea]). All AEs were non-serious, mild and classified as not related to Actrapid or devices used. One participant reported an $\mathrm{AE}$ after release from the trial site (flank pain).

\section{Cognitive performance}

On average, participants had a statistically significantly lower mean mDSST score $( \pm \mathrm{SD})$ during hypoglycaemia than euglycaemia ( $30.3 \pm 1.5$ vs $30.9 \pm 0.6$ ), with an estimated treatment difference (ETD) of -0.63 (95\% CI $-1.13,-0.14 ; p=$ 0.014) (Fig. 2a). Mean \pm SD total response time during $\mathrm{mDSST}$ and hypoglycaemia was significantly longer than during euglycaemia ( $41.5 \pm 8.9 \mathrm{~s}$ vs $38.7 \pm 7.3 \mathrm{~s})$, with an ETD of $2.86 \mathrm{~s}$ (95\% CI 0.67, 5.05; $p=0.013$ ) (Fig. 2b). Exclusion of two outliers (Fig. 2 and ESM Table 2) from the statistical analysis did not affect outcomes, with the mDSST score remaining significantly lower (ETD -0.32 [95\% CI $-0.62,-0.03], p=0.035$ ) and total response time remaining longer during hypoglycaemia compared with euglycaemia (ETD $2.00 \mathrm{~s}$ [95\% CI 0.48, 3.52], $p=0.012$ ). For working memory in the recovery phase, PASAT scores were not statistically significantly different following hypoglycaemia compared with euglycaemia, for either the $3 \mathrm{~s}$ PASAT (ETD -0.37 [95\% CI $-3.26,2.52$ ],$p=0.794$ ) or the 2 s PASAT (ETD 0.79 [95\% CI -1.34, 2.91], $p=0.454$; ESM Table 3).

\section{rCBF}

Effects of hypoglycaemia on rCBF during cDSST During the cDSST, without a working memory load, rCBF was statistically significantly lower during hypoglycaemia compared with euglycaemia in three temporal lobe regions (hippocampus, medial temporal lobe and parahippocampal gyrus) and in the striatum. In addition, $\mathrm{rCBF}$ was statistically significantly increased in six frontal lobe regions (dorsolateral prefrontal cortex, inferior frontal gyrus, middle frontal gyrus, orbitofrontal cortex, superior frontal gyrus and ventromedial prefrontal cortex), the superior parietal lobe and in the thalamus (Fig. 3a and ESM Table 4). 


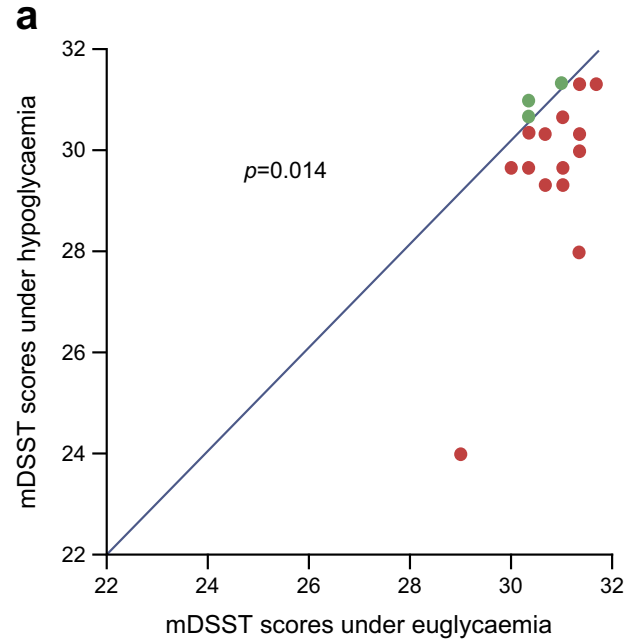

Fig. 2 (a) Observed mDSST scores and (b) response times in hypoglycaemia and euglycaemia. Only participants with available endpoints for both conditions were included in the statistical analysis. The endpoint was analysed using a linear mixed-effect model with condition (hypoglycaemia or euglycaemia) and period as fixed factors, and participant as a random factor. (a) Red dots represent participants where the correct number of mDSST responses is higher during euglycaemia than hypoglycaemia. Green dots represent participants where the correct number of mDSST responses was higher during hypoglycaemia than

\section{Effects of hypoglycaemia on rCBF during mDSST During} the mDSST (with a working memory load) rCBF was statistically significantly increased in three frontal lobe regions (dorsolateral prefrontal cortex, inferior frontal gyrus and middle frontal gyrus), the superior parietal lobe and the thalamus, and statistically significantly decreased in the hippocampus and the medial temporal lobe during hypoglycaemia (Fig. 3b and ESM Table 4).

\section{Differences in rCBF between hypoglycaemia and} euglycaemia during cDSST and mDSST Isolating working memory (measured as the rCBF during mDSST corrected for cDSST), there was statistically significantly higher blood flow in the striatum during hypoglycaemia when compared with euglycaemia (Fig. 3c), with an ETD of $0.0374 \%$ (95\% CI $0.0157 \%, 0.0590 \% ; p=0.002$ ).

\section{Hypoglycaemia assessments and counter-regulatory hormone responses}

When asked 'Do you feel hypo?' 30 min prior to induction of glycaemic conditions, the proportion of participants responding 'no' was $96.2 \%$ and $92.3 \%$, for those exposed to prior hypoglycaemia or euglycaemia, respectively. During the glycaemic clamps (nominal time $45 \mathrm{~min}$ ) proportionally more participants answered 'yes' when they were asked if they felt hypoglycaemic during hypoglycaemia $(42.3 \%)$ compared with euglycaemia $(11.5 \%)$. When we compared these subgroups in a post hoc analysis to determine the impact on the

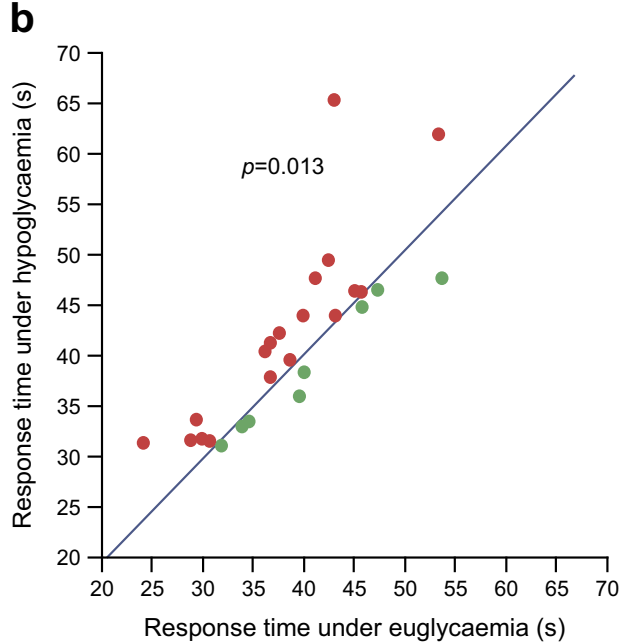

euglycaemia. ETD hypoglycaemia - euglycaemia $-0.63(95 \% \mathrm{CI}$ $-1.13,-0.14$ ). Number of correct responses (observed mean $\pm \mathrm{SD}$ : hypoglycaemia, $30.3 \pm 1.5$; euglycaemia, $30.9 \pm 0.6$ ). (b) Red dots represent participants where the mDSST response time is longer during hypoglycaemia than euglycaemia. Green dots represent participants where the mDSST response time is longer during euglycaemia than hypoglycaemia. ETD hypoglycaemia - euglycaemia $2.86(95 \% \mathrm{CI}$ $0.67,5.05$ ). Total response time (observed mean $\pm \mathrm{SD}$ : hypoglycaemia, $41.5 \pm 8.9 \mathrm{~s}$; euglycaemia, $38.7 \pm 7.3 \mathrm{~s})$

primary endpoint (mDSST score and response times), we found no differences between those responding 'yes' or 'no' to feeling the experimental hypoglycaemia (ESM Results). Mean \pm SD hypoglycaemic symptom scores 30 min prior to induction of hypoglycaemia and euglycaemia were $17.69 \pm$ 5.67 and $18.69 \pm 4.88$, respectively. Hypoglycaemic symptom scores during hypoglycaemia (55 $\mathrm{min}$ after initiating the induction of hypoglycaemia) and euglycaemia were $23.38 \pm$ 11.67 and $18.27 \pm 4.75$, respectively.

Noradrenaline, cortisol and growth hormone responses were increased during hypoglycaemia compared with euglycaemia. The response of glucagon was compromised when comparing hypoglycaemia with euglycaemia. The counter-regulatory hormonal responses are shown in Fig. 4.

\section{Discussion}

This multidisciplinary study examined how cognitive performance and its concurrent $\mathrm{rCBF}$ were affected by hypoglycaemia

Fig. 3 Normalised rCBF from PET scans and associated forest plots showing differences in brain activation in hypoglycaemia compared with euglycaemia during (a) cDSST, (b) mDSST and (c) mDSST cDSST. Only participants with available endpoints for both conditions were included in the analysis. The PET scans and forest plots are normalised to the cerebral cortex and show the percentage difference in $\mathrm{rCBF}$ in hypoglycaemia compared with euglycaemia (green, increase in $\mathrm{rCBF}$; red, decrease in $\mathrm{rCBF}$ ). Endpoints were compared using an ANOVA model with condition, period and participant as fixed factors 


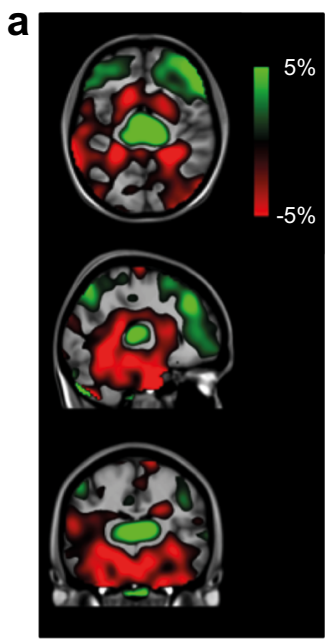

Thalamus Frontal lobe Dorsolateral prefrontal cortex Inferior frontal gyrus Middle frontal gyrus

Orbitofrontal cortex

Superior frontal gyrus

Ventromedial prefrontal cortex Parietal lobe

Superior parietal lobe Posterior supramarginal gyrus

Precuneus temporo-parietal Occipital lobe

Primary visual cortex Limbic lobe Anterior cingulate gyrus/cortex Posterior cingulate gyrus Temporal lobe Hippocampus

Insula temporal lobe

Medial temporal lobe Parahippocampal gyrus Basal ganglia Globus pallidus
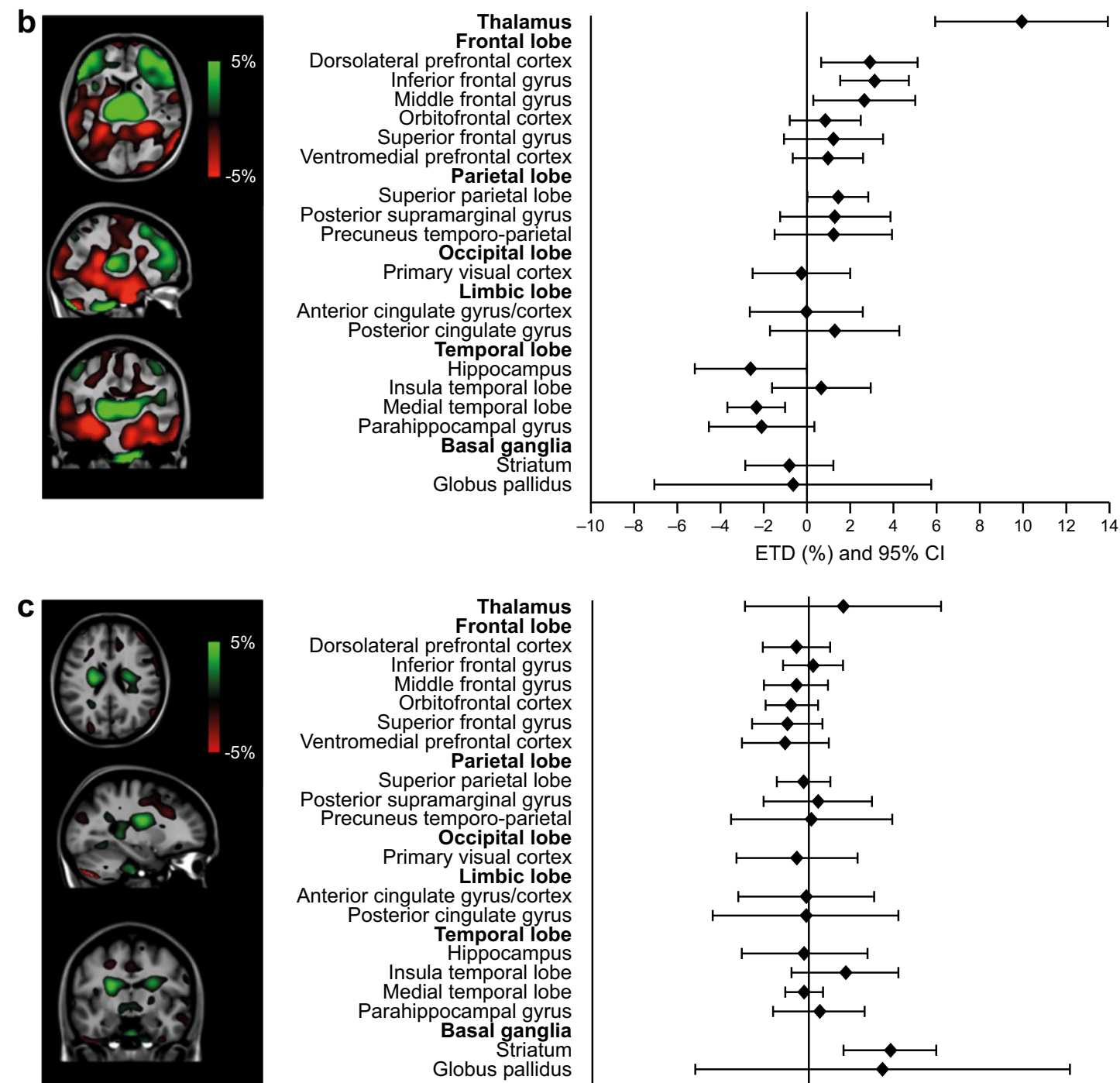

Thalamus Frontal lobe Dorsolateral prefrontal cortex Inferior frontal gyrus

Middle frontal gyrus

Orbitofrontal cortex

Orbitofrontal cortex
Superior frontal gyrus Ventromedial prefrontal cortex Parietal lobe

Superior parietal lobe

Posterior supramarginal gyrus Precuneus temporo-parietal Occipital lobe

Primary visual cortex Limbic lobe

Anterior cingulate gyrus/cortex Posterior cingulate gyrus Temporal lobe Hippocampus

nsula temporal lobe Medial temporal lobe

Parahippocampal gyrus Basal ganglia Globus pallidus
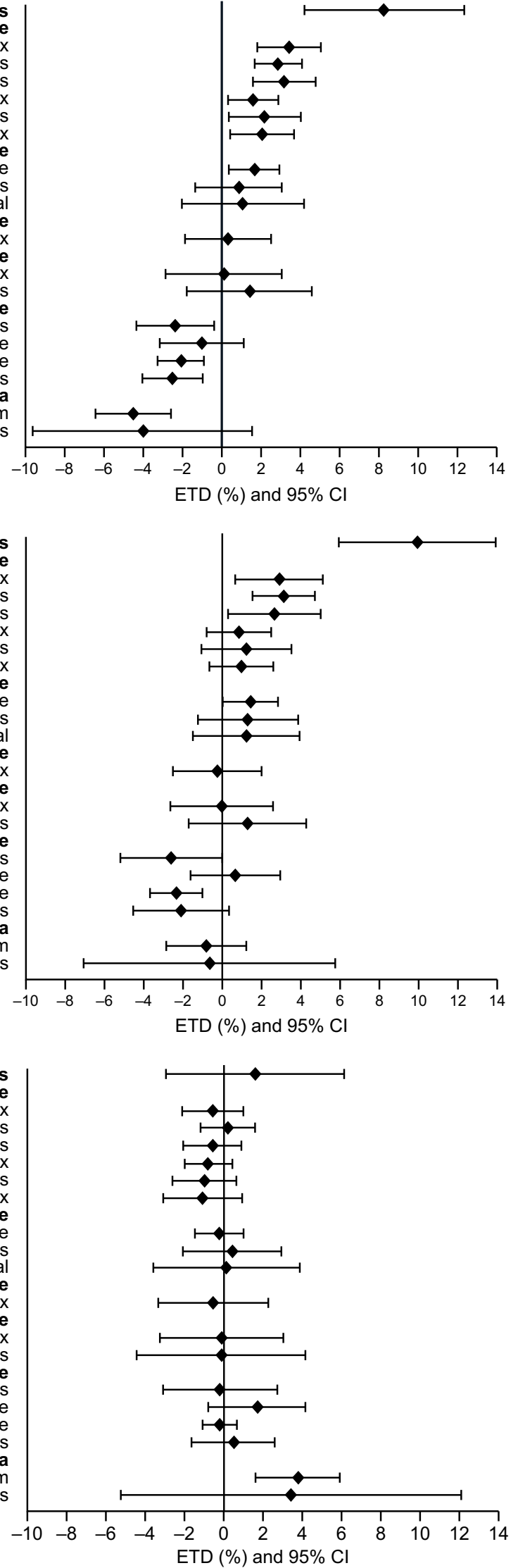
Fig. 4 Counter-regulatory hormonal responses for (a) noradrenaline, (b) glucagon, (c) cortisol and (d) growth hormone. Analysis was based on completers. Solid line represents hypoglycaemia; dashed line represents euglycaemia a

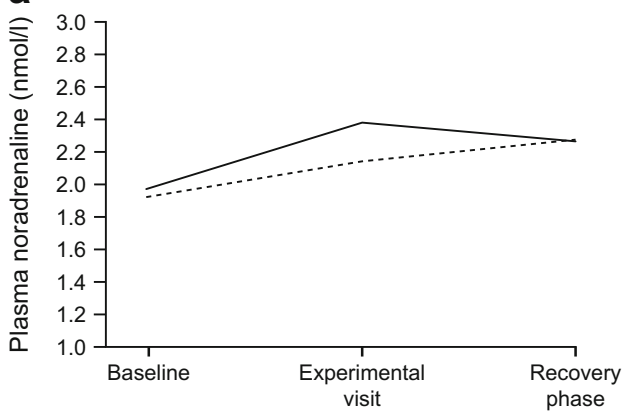

C

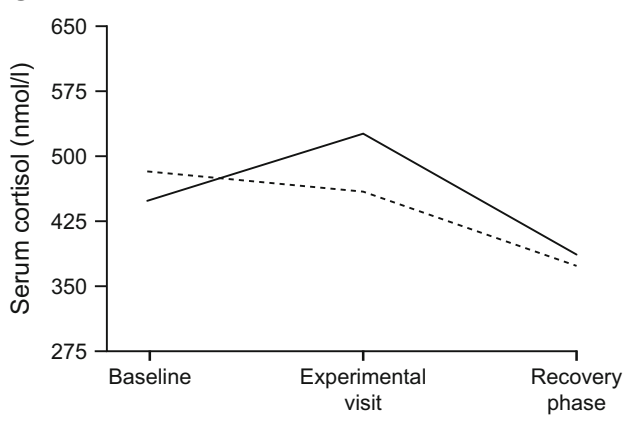

b
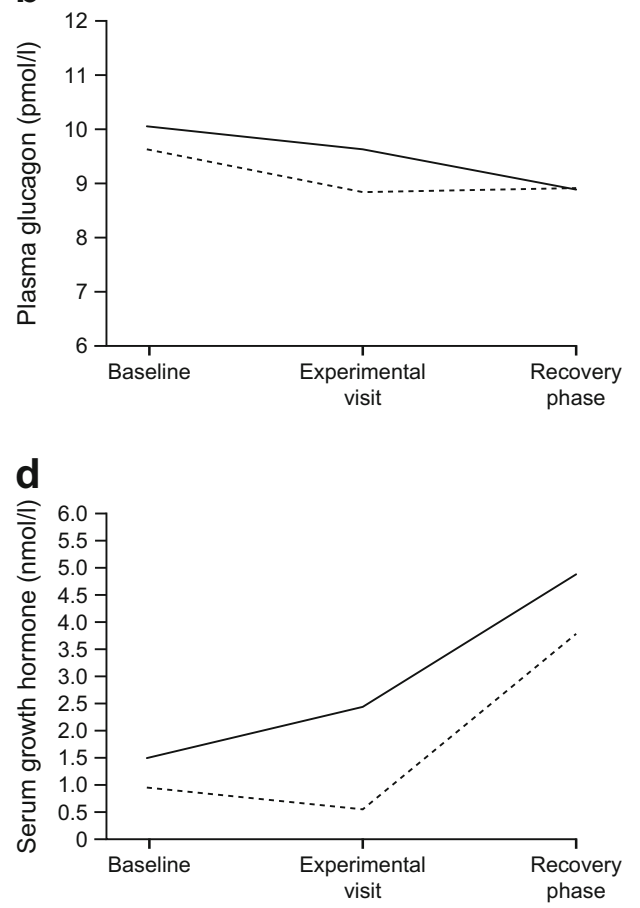

at a mean PG of $2.9 \mathrm{mmol} / \mathrm{l}$. This is a higher level of PG than has been tested in previous studies [1,2], but matches the level recommended in reporting of clinical trials by the recent position statement from the International Hypoglycaemia Study Group [35] and is considered to be 'sufficiently low to indicate serious, clinically important hypoglycaemia'. During hypoglycaemia there was a modest, yet significant, decrease in the number of correct $\mathrm{mDSST}$ responses referring to working memory, as well as a significant increase in response time. The effects of hypoglycaemia on $\mathrm{rCBF}$ in the striatum was statistically significant, highly uniform and with little variation (in SD) between participants. Furthermore, use of working memory during hypoglycaemia was associated with a significant increase in blood flow in the striatum.

Previous studies have reported reductions in cognitive function scores in the range of $2-40 \%$ during hypoglycaemia $[8,36]$. The comparatively small decrease in number of correct $\mathrm{mDSST}$ responses in our study indicates, as expected, that working memory function was relatively preserved with regard to accuracy but at the cost of response speed [3]. These results indicate that during hypoglycaemia, at a mean PG of $2.9 \mathrm{mmol} / \mathrm{l}$, participants with type 1 diabetes mellitus may be able to keep performance at a similar level as during euglycaemia, at least when performing less complex tasks, but at a $7 \%$ slower execution rate $(3 \mathrm{~s}$ over a block of 32 questions). While this level of slowing may not be of great importance for many daily functions where response time is not critical, it could become serious in tasks which do depend on rapid information processing, such as driving.
Our study showed no difference in the number of correct PASAT responses in the recovery phase following hypoglycaemia compared with euglycaemia, possibly reflecting time of testing (75-90 min after euglycaemia restoration). Cognitive performance during recovery from hypoglycaemia has varied between different studies [4-6]. In one study, the cognitive tests were repeated at 10-15 min intervals up to $85 \mathrm{~min}$ after restoration of euglycaemia, and cognitive performance, for some tasks, was found to be only impaired for up to $10 \mathrm{~min}$ after euglycaemia was restored [6].

During the hypoglycaemic clamp, approximately $58 \%$ of participants answered 'no' to feeling hypoglycaemic, despite efforts made to exclude participants with impaired awareness of hypoglycaemia. Despite this, rCBF was significantly impacted during both cDSST and mDSST tasks. Indeed, both were associated with increased $\mathrm{rCBF}$ in frontal lobe regions (goal-directed action, behavioural control and problem solving) [34, 37] and the thalamus (relay station) and decreased rCBF in temporal lobe regions (memory functions). Furthermore, some regions that were deactivated during cDSST (and hence received reduced $\mathrm{rCBF}$ ) were less affected during $\mathrm{mDSST}$ when working memory function was required; hence, $\mathrm{rCBF}$ was also higher in the basal ganglia and insula.

The effects of hypoglycaemia on rCBF in the thalamus, insula and globus pallidus were not statistically significantly different between cDSST and mDSST, but the effect in the striatum was significantly different and was highly uniform across participants, as indicated by the narrow confidence intervals (Fig. 3c). 
In people with type 1 diabetes mellitus, studies have revealed increased $\mathrm{CBF}$ in the hypothalamus, brainstem, anterior cingulate cortex and putamen when reducing PG from 5.2 to $4.3 \mathrm{mmol} / \mathrm{l}$. This suggests that these regions are sensitive to small blood glucose changes, and that even small sudden glycaemic changes may be of clinical importance [11]. In the current study, increased $\mathrm{rCBF}$ in the frontal lobe areas during hypoglycaemia could reflect the processing of input from the thalamus, which was also highly activated. Working memory was associated with increased blood flow in the striatum, a part of the basal ganglia thought to support motor functions, memory, language and emotion regulation. Increased $\mathrm{rCBF}$ in this region may suggest an increased support of this subcortical system during a task that is otherwise related to frontal regions, suggesting that the brain requires more resources to maintain performance during hypoglycaemia. A less efficient brain (i.e. recruiting more resources to preserve cognitive performance) during hypoglycaemia ( $\mathrm{PG} \leq 2.8 \mathrm{mmol} / \mathrm{l})$ in individuals with type 1 diabetes mellitus was also shown by a previous study that used a working memory task during fMRI [9].

\section{Study limitations}

Regional blood flow averages did not allow testing for significant changes of blood flow in smaller parts of individual brain regions; this would require specific voxel-based analysis. A further limitation is that individual responses to hypoglycaemia can vary widely [38], as illustrated here by the finding that about half of participants had an impaired awareness of hypoglycaemia during the clamp and thus did not feel the impact of hypoglycaemia during the hypoglycaemic clamp. This may be attributed to the milder levels of hypoglycaemia achieved in the study (PG $2.9 \mathrm{mmol} / \mathrm{l}$ ) and the comparable threshold for appearance of symptoms [39, 40]; however, it may also reflect the inclusion of participants with previously unrecognised unawareness and an inability to identify reduced awareness in everyday life. A more complete picture of potential impairments in counter-regulatory hormonal responses would have been afforded if adrenaline (epinephrine) had also been measured. The cDSST and mDSST tasks were not alternated and therefore the $\mathrm{rCBF}$ response to hypoglycaemia may be subject to time-dependent differences in activation. Although a time-dependent impact of hypoglycaemia on the brain has been suggested [29], $\mathrm{H}_{2}{ }^{15} \mathrm{O}$ PET imaging for the cDSST and mDSST tasks commenced after the target hypoglycaemic PG was reached (0-20 min and 30$60 \mathrm{~min}$, respectively). Therefore, effects of decreasing PG on the brain were not captured in this study and will limit any potential time-dependent effect. Finally, no cognitive dysfunction screening was carried out on participants prior to the study; however, cognitive problems related to working memory or other memory functions are not typically seen in adults with type 1 diabetes mellitus [41]. Despite these limitations the data still challenge the prevailing notion that working memory is not impaired until glucose levels are $<2.8 \mathrm{mmol} / \mathrm{l}$ and shows that working memory is also impaired between 2.8 and $3.0 \mathrm{mmol} / \mathrm{l}$, the glucose levels that generally reflect the lower and upper limits for the manifestation of hypoglycaemia symptoms [39, 40].

In conclusion, this hypothesis-driven study demonstrated that working memory performance and reaction times are adversely affected by hypoglycaemia in individuals with type 1 diabetes mellitus, and that they are associated with measurable effects on rCBF, even at mean PG concentrations of $2.9 \mathrm{mmol} / 1$. The findings are clinically relevant, supporting the importance of reducing the risk of hypoglycaemic episodes at this level.

Acknowledgements The expert technical assistance of A. Mengel, L. Egefjord, M. Bohl, J. Larsen, K. Vang, U. Sigsgaard, L.B. Vad, D.G. Zeidler and the staff at PET Center and Department of Anesthesiology, Aarhus University Hospital, Aarhus, Denmark is gratefully acknowledged.

The authors thank the trial staff and participants. They also thank C. Rosano (Department of Epidemiology, University of Pittsburgh, PA, USA) and A. Howard (School of Medicine and Department of Bioengineering, University of Pittsburgh, PA, USA) for providing the DSST tests, and C. Ryan (Department of Psychiatry, University of Pittsburgh, PA, USA) for his valuable help interpreting the DSST results in the context of existing literature. The authors thank M. Gudmundsson, K. Kaplan and E. HachmannNielsen (Novo Nordisk, Søborg, Denmark) for assistance with article preparation. The authors acknowledge medical writing and submission support provided by S. Mason, L. Brackenbury, G.F. Hansa-Wilkinson and D. Renshaw of Watermeadow Medical, an Ashfield company, part of UDG Healthcare. This support was funded by Novo Nordisk. Finally, N. Møller (Aarhus University Hospital, Aarhus, Denmark) is thanked for invaluable guidance regarding the clamp procedures.

Data availability The data generated during and/or analysed during the current trial are available from the corresponding author on reasonable request.

Funding This study was funded by Novo Nordisk. Novo Nordisk was involved in the trial design and protocol development, provided logistical support, and obtained the data, which were evaluated jointly by the authors and the sponsor. All authors interpreted the data and wrote the manuscript together with the sponsor's medical writing services. The authors had the final decision to approve and submit the manuscript for publication. M. Gejl was financially supported by the Department of Biomedicine, Aarhus University, Aarhus, Denmark, and supported by grants of the Anniversary Fund for doctors and Health Research Fund of Central Denmark Region. E. van Duinkerken was supported by a grant of the Brazilian National Council for Scientific and Technological Development (CNPq).

Duality of interest EvD reports consultancy for Novo Nordisk. HLH, CTH and KLSP are employees and shareholders at Novo Nordisk. PLC was an employee and stakeholder of Novo Nordisk throughout the duration of the study. MG, AG, BB, AM and JR declare that there is no duality of interest associated with their contribution to this manuscript.

Contribution statement MG, BB and JR contributed to the trial design, participant recruitment, experiments, data interpretation, discussion of the results, and review and editing of the manuscript. PLC contributed to the statistical analyses, and review and editing of the manuscript. AG researched and analysed data, and revised the manuscript. AM contributed to the trial design, experiments, and revised the manuscript. KLSP, HLH and $\mathrm{CTH}$ contributed to the trial design, data interpretation, discussion of the results, and review and editing of the manuscript. EvD adapted the working memory test for use in the PET scanner, contributed to the interpretation of the results, and revised the manuscript. All authors provided approval of the version to be published. JR is the guarantor of this work 
and, as such, had full access to all the data in the study and takes responsibility for the integrity of the data and the accuracy of the data analysis.

Open Access This article is distributed under the terms of the Creative Commons Attribution 4.0 International License (http:// creativecommons.org/licenses/by/4.0/), which permits unrestricted use, distribution, and reproduction in any medium, provided you give appropriate credit to the original author(s) and the source, provide a link to the Creative Commons license, and indicate if changes were made.

\section{References}

1. Allen KV, Pickering MJ, Zammitt NN et al (2015) Effects of acute hypoglycemia on working memory and language processing in adults with and without type 1 diabetes. Diabetes Care 38:1108-1115

2. Graveling AJ, Deary IJ, Frier BM (2013) Acute hypoglycemia impairs executive cognitive function in adults with and without type 1 diabetes. Diabetes Care 36:3240-3246

3. Inkster B, Frier BM (2012) The effects of acute hypoglycaemia on cognitive function in type 1 diabetes. Br J Diabetes Vasc Dis 12:221-226

4. Evans ML, Pernet A, Lomas J, Jones J, Amiel SA (2000) Delay in onset of awareness of acute hypoglycemia and of restoration of cognitive performance during recovery. Diabetes Care 23:893-897

5. Gold AE, MacLeod KM, Deary IJ, Frier BM (1995) Hypoglycemia-induced cognitive dysfunction in diabetes mellitus: effect of hypoglycemia unawareness. Physiol Behav 58:501-511

6. Zammitt NN, Warren RE, Deary IJ, Frier BM (2008) Delayed recovery of cognitive function following hypoglycemia in adults with type 1 diabetes: effect of impaired awareness of hypoglycemia. Diabetes 57:732-736

7. Blackman JD, Towle VL, Sturis J, Flewis G, Spire J-P, Polonsky KS (1992) Hypoglycemic thresholds for cognitive dysfunction in IDDM. Diabetes 41:392

8. Gonder-Frederick LA, Cox DJ, Driesen NR, Ryan CM, Clarke WL (1994) Individual differences in neurobehavioral disruption during mild and moderate hypoglycemia in adults with IDDM. Diabetes 43:1407-1412

9. Bolo NR, Musen G, Jacobson AM et al (2011) Brain activation during working memory is altered in patients with type 1 diabetes during hypoglycemia. Diabetes 60:3256-3264

10. Mangia S, Tesfaye N, De Martino F et al (2012) Hypoglycemiainduced increases in thalamic cerebral blood flow are blunted in subjects with type 1 diabetes and hypoglycemia unawareness. J Cereb Blood Flow Metab 32:2084-2090

11. Page KA, Arora J, Qiu M, Relwani R, Constable RT, Sherwin RS (2009) Small decrements in systemic glucose provoke increases in hypothalamic blood flow prior to the release of counterregulatory hormones. Diabetes 58:448-452

12. Vafaee MS, Vang K, Bergersen LH, Gjedde A (2012) Oxygen consumption and blood flow coupling in human motor cortex during intense finger tapping: implication for a role of lactate. J Cereb Blood Flow Metab 32:1859-1868

13. van Golen LW, Huisman MC, Ijzerman RG et al (2013) Cerebral blood flow and glucose metabolism measured with positron emission tomography are decreased in human type 1 diabetes. Diabetes 62:2898-2904

14. Kameyama M, Murakami K, Jinzaki M (2016) Comparison of [(15)O] $\mathrm{H}(2) \mathrm{O}$ positron emission tomography and functional magnetic resonance imaging in activation studies. World J Nucl Med 15:3-6

15. International Conference on Harmonisation of Technical Requirements for Registration of Pharmaceuticals for Human Use (1996) ICH harmonised tripartite guideline: guideline for good clinical practice E6 (R1). International Conference on Harmonisation, Geneva
16. World Medical Association (2013) WMA Declaration of Helsinki Ethical Principles for Medical Research Involving Human Subjects. 64th WMA General Assembly, October 2013, Fortaleza, Brazil

17. Wechsler D (1981) WAIS-R manual. The Psychological Corporation, New York

18. Salthouse TA, Fristoe N, Rhee SH (1996) How localized are agerelated effects on neuropsychological measures? Neuropsychology 10:272-285

19. Venkatraman VK, Aizenstein H, Guralnik J et al (2010) Executive control function, brain activation and white matter hyperintensities in older adults. NeuroImage 49:3436-3442

20. Gronwall DM (1977) Paced auditory serial-addition task: a measure of recovery from concussion. Percept Mot Skills 44:367-373

21. Schachinger H, Cox D, Linder L, Brody S, Keller U (2003) Cognitive and psychomotor function in hypoglycemia: response error patterns and retest reliability. Pharmacol Biochem Behav 75:915-920

22. Deary IJ, Hepburn DA, MacLeod KM, Frier BM (1993) Partitioning the symptoms of hypoglycaemia using multi-sample confirmatory factor analysis. Diabetologia 36:771-777

23. Kannurpatti SS, Motes MA, Rypma B, Biswal BB (2010) Neural and vascular variability and the fMRI-BOLD response in normal aging. Magn Reson Imaging 28:466-476

24. Nakahachi T, Ishii R, Iwase $\mathrm{M}$ et al (2008) Frontal activity during the digit symbol substitution test determined by multichannel nearinfrared spectroscopy. Neuropsychobiology 57:151-158

25. Usui N, Haji T, Maruyama M et al (2009) Cortical areas related to performance of WAIS Digit Symbol Test: a functional imaging study. Neurosci Lett 463:1-5

26. Bie-Olsen LG, Kjaer TW, Pedersen-Bjergaard U et al (2009) Changes of cognition and regional cerebral activity during acute hypoglycemia in normal subjects: $\mathrm{a}_{2}{ }^{15} \mathrm{O}$ positron emission tomographic study. J Neurosci Res 87:1922-1928

27. Rosenthal JM, Amiel SA, Yaguez L et al (2001) The effect of acute hypoglycemia on brain function and activation: a functional magnetic resonance imaging study. Diabetes 50:1618-1626

28. Schafer RJ, Page KA, Arora J, Sherwin R, Constable RT (2012) BOLD response to semantic and syntactic processing during hypoglycemia is load-dependent. Brain Lang 120:1-14

29. Teh MM, Dunn JT, Choudhary P et al (2010) Evolution and resolution of human brain perfusion responses to the stress of induced hypoglycemia. NeuroImage 53:584-592

30. Teves D, Videen TO, Cryer PE, Powers WJ (2004) Activation of human medial prefrontal cortex during autonomic responses to hypoglycemia. Proc Natl Acad Sci U S A 101:6217-6221

31. Watanabe Y, Funahashi S (2012) Thalamic mediodorsal nucleus and working memory. Neurosci Biobehav Rev 36:134-142

32. Yee LT, Roe K, Courtney SM (2010) Selective involvement of superior frontal cortex during working memory for shapes. J Neurophysiol 103: $557-563$

33. Zarahn E, Rakitin B, Abela D, Flynn J, Stern Y (2005) Positive evidence against human hippocampal involvement in working memory maintenance of familiar stimuli. Cereb Cortex 15:303-316

34. van Duinkerken E, Schoonheim MM, Steenwijk MD et al (2014) Ventral striatum, but not cortical volume loss, is related to cognitive dysfunction in type 1 diabetic patients with and without microangiopathy. Diabetes Care 37:2483-2490

35. International Hypoglycaemia Study Group (2017) Glucose concentrations of less than $3.0 \mathrm{mmol} / \mathrm{l}(54 \mathrm{mg} / \mathrm{dl})$ should be reported in clinical trials: a joint position statement of the American Diabetes Association and the European Association for the Study of Diabetes. Diabetologia 60:3-6

36. Mitrakou A, Ryan C, Veneman T et al (1991) Hierarchy of glycemic thresholds for counterregulatory hormone secretion, symptoms, and cerebral dysfunction. Am J Phys 260:E67-E74

37. Stuss DT (2011) Functions of the frontal lobes: relation to executive functions. J Int Neuropsychol Soc 17:759-765 
38. Wyatt JP, Illingworth RN, Graham CA, Hogg K (2012) Oxford handbook of emergency medicine. Oxford University Press, Oxford

39. Kenny C (2014) When hypoglycemia is not obvious: diagnosing and treating under-recognized and undisclosed hypoglycemia. Prim Care Diabetes 8:3-11
40. Sprague JE, Arbelaez AM (2011) Glucose counterregulatory responses to hypoglycemia. Pediatr Endocrinol Rev 9:463-473

41. Brands AM, Biessels GJ, de Haan EH, Kappelle LJ, Kessels RP (2005) The effects of type 1 diabetes on cognitive performance: a meta-analysis. Diabetes Care 28:726-735 